\title{
Microwaving biological cells - Intracellular analysis with microwave dielectric spectroscopy
}

Authors: François Artis, Tong Chen, Thomas Chrétiennot, Jean-Jacques Fournié, Mary Poupot, David Dubuc, Katia Grenier

François Artis, David Dubuc (dubuc@laas.fr), Katia Grenier (grenier@laas.fr) are with LAAS-CNRS, Toulouse, France.

Tong Chen, Thomas Chrétiennot were formerly with LAAS-CNRS, Toulouse, France.

J-J. Fournié and M. Poupot (m.poupot@inserm.fr) are with the Cancer Research Center in Toulouse, France

Probing biological materials with electromagnetic waves has been investigated for decades [1]-[6]. It consists in characterizing the sample with its dielectric properties, which are frequencydependent. Dielectric spectroscopy is performed by placing a high-frequency circuit close to the biological species under study. Passing through the biological medium, the electric properties of the biological elements modify the electromagnetic field. The complex permittivity of the medium under test may therefore be extracted and provides a dielectric signature of the sample.

Past investigations were mainly performed at the organ and tissue levels, with large quantities of liquids. They provided essential knowledge about the dielectric properties of biological matter and led to key applications such as hyperthermia and cancer ablation [3] and more recently to microwave cancer imaging [7]-[8] due to the possible discrimination of tumorous and healthy tissues [9]-[11]. Today, with the extensive development of micro and nanotechnologies in conjunction with the frequency increase of electromagnetic instruments, a dramatic miniaturization of RF circuits has ensued leading to sizes compatible with cells and virus dimensions (just a few micrometers in less than microliters) with frequency in the microwave and millimeter wave domains. Thus the dielectric characterization of the biological elements may be carried out at the molecular and cellular levels. Access to miniature cellular and molecular characterization paves the way for greater insights and a better understanding of the living and its interaction with electromagnetic waves as well as innovative applications. Cell observation is indeed a routine technique for biological and medical investigations.

Traditionally cell analysis has relied on optical detection systems. Microscopy using stains or fluorescent dyes and flow cytometry are the main techniques used due to their high efficiency and specificity. These techniques have been highly successful over the last two decades. This is because people, and more particularly biologists and physicians, naturally tend to trust images since it involves eyesight, which we all use everyday. On the contrary electromagnetic waves and permittivity are not so easily perceived as these notions do not refer to our senses and remain conceptually harder to grasp. Moreover, optical techniques may be very efficient and precise due to the fluorochromes and the antibodies used for attachment in the cells. Intracellular microscopy even enables the measurement of multiple intracellular markers simultaneously at the single cell level. An example of an image obtained is shown in Figure 1 [12]. Three dyes have been used to highlight three major constituents of eukaryote cells: nucleus in blue, actin in green (notably present in the cytoskeleton) and mitochondria in red. This snapshot is therefore information-rich. The precision obtained to determine each cell and constitutive part, as highlighted by their specific stain, is absolutely excellent. Real time monitoring of biological reactions is even accessible during the fluorochromes emission time. 


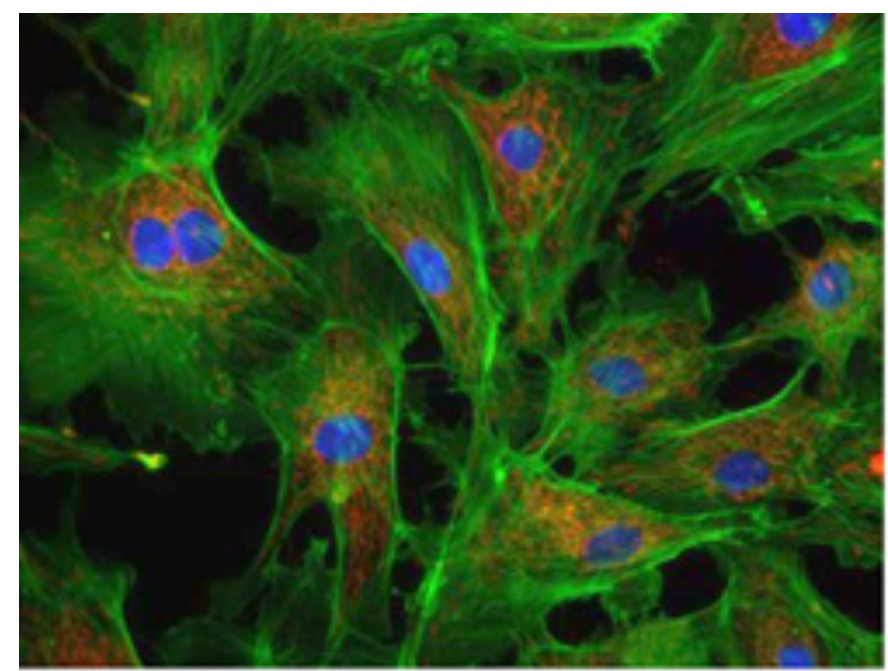

Figure 1. Example of an image of cells stained with three dyes highlighting nucleus (b/ue), actin (green), and mitochondria (red) [12].

However, the required equipment is often costly, and the technique may be considered invasive for the investigated cells. It chiefly involves labeling techniques, through various stains and fluorochromes, which interact on the surface or inside the cells. Fluorophores are preliminary attached to an antibody. When the latter recognizes its corresponding antigen present on or inside the cell, binding between the antigen and antibody occurs and permits the expression of the fluorophore when excited with the adequate wavelength applied with a UV lamp or laser. These labels may therefore induce cell modifications, leading to variations in the mechanism to be detected Erroneous or unwanted observations may result. Also, in the case of intracellular staining, cells must first be fixed and permeabilized, stained, and then analyzed by flow cytometry or microscopy. These steps are not only time-consuming, but also destructive for the cells. Further analysis may not be performed on the exact same cells. The development of new biological analysis instruments at the cellular and molecular levels, which would be either noninvasive or without induced perturbation, is therefore highly challenging and would be particularly interesting in various applications.

In the meantime, the biological and medical domains are submitted to massive modifications with the dramatic development of microfluidics [13]-[14], corresponding to the appropriate size to reach biomolecules and cells properties. At present, micro- and nano-technologies offer wellcontrolled dimensions and fluidic volumes (as low as the $\mu$ liter or even smaller) at quite a low cost and high fabrication volumes without the drawback of manual handling. It is now possible to reproducibly engineer the cell's microenvironment at a cellular resolution and at a low cost whilst mimicking complex biochemistries (cells interaction, gradients, cells contacts...). Cell culture, sorting, handling and transportation [15], as well as addition of chemicals, combined with single to arrays arrangements for high throughput analysis [16]-[19] have been demonstrated. One important bottleneck of this micrometric integration lies in the miniaturization of suitable analyzing systems. The main issue is that of putting forward integrated characterization techniques that are both sensitive and non-invasive in a liquid medium. Extensive work is underway to address these issues. Among the developed miniature bio-sensing methods [20], such as optical, electrical and mechanical analysis [21]-[28], impedancemetry using microwaves and millimeterwaves stands out as a promising candidate. The technique does present interesting features, which will be detailed in this paper.

\section{Why microwaves?}

Miniature microwave dielectric spectroscopy resulted in several breakthroughs in biological investigations, particularly inmolecular and cellular analysis.

\section{Frequency dependence of permittivity}


During the past century Pr. Schwan [1]-[2] demonstrated that the interaction of the biological matter with electromagnetic waves is frequency-dependent and translates into relaxations and resonances of the complex permittivity. These permittivity and conductivity dispersions are related to different polarization phenomena depicted in Fig. 2.

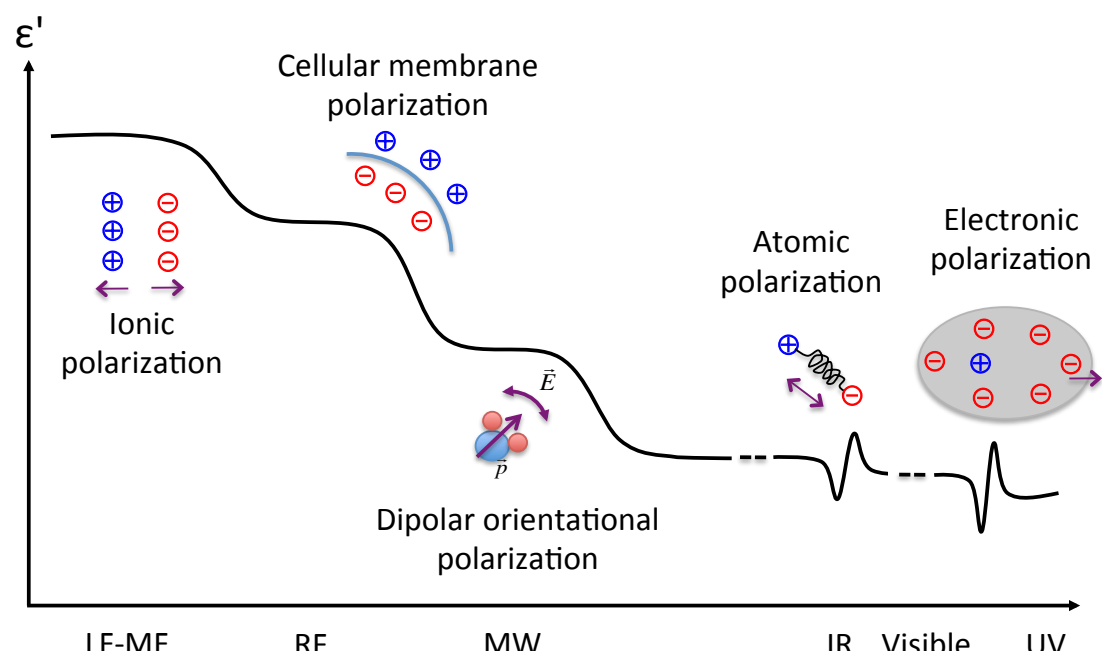

Frequency

Figure 2. Relative dielectric constant versus frequency with related polarization mechanisms in biological matter.

The first dispersion, named $\alpha$ and occurring in the low frequency range, is associated with ionic diffusion. The $\beta$ dispersion, located in the $\mathrm{MHz}$ range, is related to polarization of the cellular membrane with the charging of the capacitive cell membrane. Its exploitation during the past decade with the development of low frequency impedance-based cytometry [24]-[26] has led to impressive biological detection capabilities based on membrane polarization, with the discrimination of white blood cells (lymphocytes, neutrophils, monocytes) [Holmes 2009]. Thus it became possible to gain insights into the viability, size and morphology of cells. However, in terms of discrimination, the technique remains fairly limited in the case of cells exhibiting the same size and morphology. Membrane permeabilization is then required to enable cell penetration to the electrical field. To extend the analyzing potential, increasing the frequency range to the $\mathrm{GHz}$ regime has become an attractive solution.

\section{Intracellular probing and monitoring}

By rising the frequency, the capacitive barrier of the cell membrane may be crossed and waves may penetrate into the cells, as shown in Figure 3 . This enhances the interaction of the electromagnetic waves with the biological matter. It is associated with the $\mathrm{y}$ dispersion due to the polarization of dipoles, occurring in the microwave and millimeterwave ranges and due to the molecular reorientation dynamics. The relaxation phenomenon is common to a large variety of aqueous solutions of bio-species and bio elements. The most well-known is the dielectric relaxation induced by water molecules around $20 \mathrm{GHz}$, which is a large constituent of the living. Further discrimination and observation of biological mechanisms may therefore be expected with microwave spectroscopy. Beyond, in the $\mathrm{THz}$ regime, other mechanisms also develop based on vibrational modes and atomic variations [29]. 


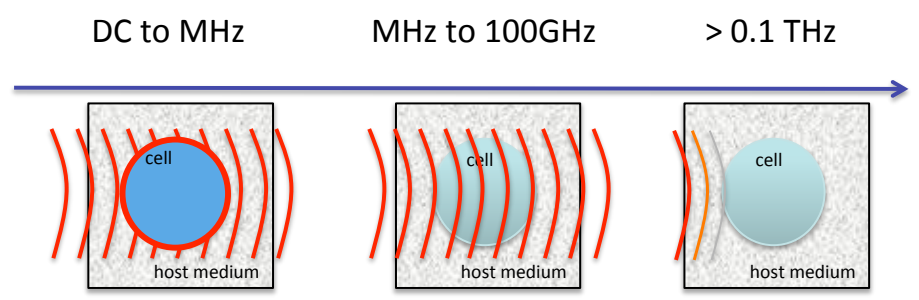

Figure 3. Frequency range impact on electromagnetic wave penetration in liquid media.

\section{Noninvasive and highly miniaturizable}

Moreover, microwave dielectric spectroscopy offers the advantage of being contactless, nondestructive and label-free. Possible contamination or perturbation of the bio-material (e.g., living cells) is suppressed during characterization. Due to the frequency range (microwave and millimeterwave), the RF circuits feature high integration with the use of micro-nanotechnologies, which makes the sensing technique compatible with all lab-on-a-chip developments. It therefore facilitates cells manipulation and preparation, like sorting, or integration of biological protocols with microfluidic networks. The detection area may be easily sized to the cell dimensions or to ultra small volumes of biological samples (microliters and below).

The sensing technique is noninvasive for cells under low power to avoid any interference with the living. Thus cells can be kept alive during tests, thereby promoting real time monitoring of biological reactions. Due to wave penetration into the cells, one might expect access to intracellular events or modifications. However this is not the case with low frequency techniques limited to cell membrane observation (see Figure 3).

\section{Compatible with ionic content of biological media}

Another important feature of microwave spectroscopy corresponds to its complete adequacy with rich biological culture media. Those liquids contain indeed salt (typical value of $9 \mathrm{gr} / \mathrm{l}$ ), ions and nutrients, which below $1 \mathrm{GHz}$ induce a large ionic conductivity and losses. Electrode polarization also occurs with low frequency and DC methods. It has then to be taken into account by calculation, whereas media at low frequency may also be depleted. On the contrary, living cells may be directly studied in their traditional and rich media in the microwave range.

\section{Dielectric signature and selectivity}

The next breakthrough corresponds to the richness of the dielectric spectra (real and imaginary part of the complex permittivity versus frequency). Gabriel et al. [30] reviewed and evaluated the dielectric properties of various biological samples such as blood, bone, fat and grey matter. Distinct dielectric spectra were obtained and highlighted a definite specificity of the dielectric properties, making up a "dielectric signature". This has also been demonstrated with molecules [31][32]. An example of this type of signature at the molecular level is given in Figure 4 [33]. It depicts the real and imaginary permittivity spectra of two biomolecules in aqueous solutions, saccharose and albumin, exhibiting the same mass concentration of $100 \mathrm{mg} / \mathrm{ml}$. 


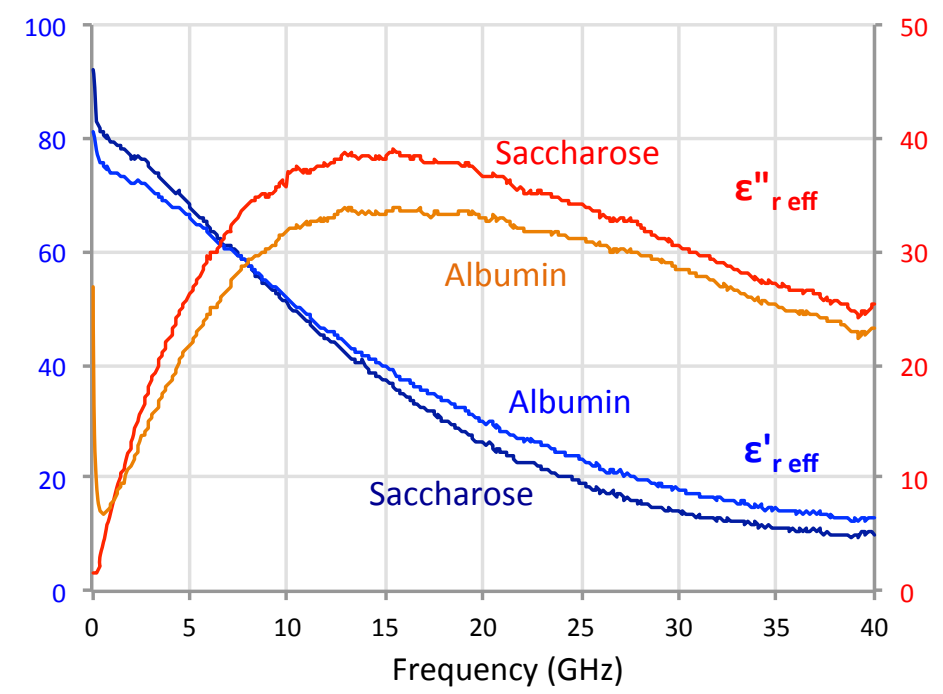

Figure 4. Dielectric spectra of saccharose and albumin in aqueous solution with a concentration of $100 \mathrm{mg} / \mathrm{ml}[33]$.

Due to their different dipolar moment, size and composition, their respective molecular dynamic is distinctive and leads to singular dielectric properties. Applied to a wide variety of biomolecules, such as aminoacids and carbohydrates, a mapping of the dielectric properties of the biomolecules has been drawn up at a single frequency [33]. This dielectric mapping supports molecule discrimination and could be applied to cells and tissues. However, mentioning a dielectric specificity of all biological elements would be far-fetched and abusive. Limitations have to be defined. Similar to low frequency (in the $\mathrm{MHz}$ range) detection, which may not differentiate cells with similar size and morphology, biological materials may exhibit analogous dielectric properties in the $\mathrm{GHz}$ domain. Further discrimination than in the $\mathrm{MHz}$ regimen is nevertheless expected, especially at the cellular level, due to wave penetration into cells.

Consequently, all these features may be seen as a great opportunity to detect and characterize cells based on their content as microwaves and millimeterwaves may penetrate into them. The possible detection and discrimination of the cells' pathological state, morphology and type is therefore expected. However this calls for intensive development in terms of circuits design for enhanced sensitivity, biocompatible technologies, characterization and modeling works in order to build the link between biophysical states and electromagnetic signatures.

\section{Cellular microwave-based biosensors: for cell population down to single-cell analysis}

The development of miniature microwave biosensors for cellular and molecular investigations is a recent activity, which requires defining and demonstrating the technique's potential. Stuchly et al., first initiated it in 1998 [34] introducing a coplanar sensor configuration with a reservoir placed on top for liquid placement, followed by Facer et al., in 2001 [35] with the measurements of hemoglobin, DNA molecules and E-coli bacteria in solutions and by Anghyun et al., in 2008 with permittivity extraction of human embryonic kidney cells (HEK-293) in DMEM medium [36]. Configurations implementing a microfluidic channel have emerged [37]-[40] to investigate cells in their liquid environment, as well as resonant topologies with cells blocked in a gel [41].

So far several demonstrations have been achieved, such as the ability to detect the living cells in their biological medium with adequate sensitivity and selectivity in particular. This has been performed with cell suspensions exhibiting large concentrations in the order of several millions of cells per milliliter [38]-[39], down to the single cell with different microwave biosensors [42], as shown in Fig. 5. 
For millions of cells $/ \mathrm{ml}$

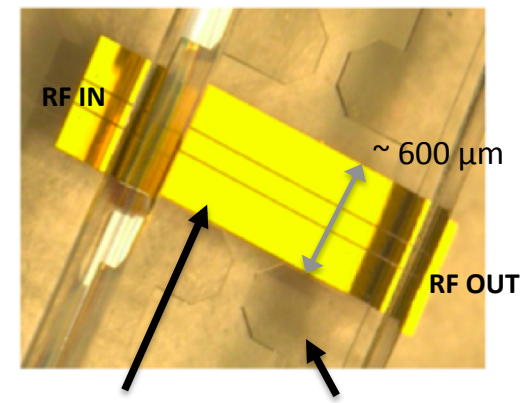

Fluid under test Microfluidic channel
For tens of cells

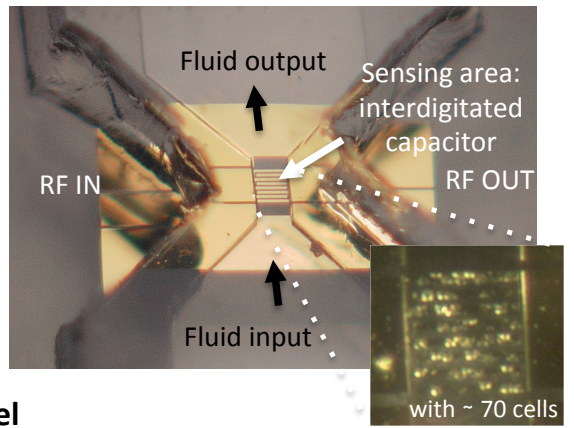

For single cell analysis

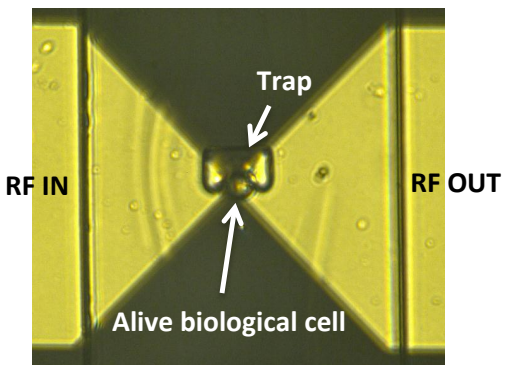

Figure 5. Miniature fluidic and microwave biosensors based on a coplanar waveguide, an interdigitated capacitor [33] and a capacitive gap [42] configurations for millions of cells $/ \mathrm{ml}$ down to single-cell analysis.

As mentioned previously with respect to the different breakthroughs in microwave dielectric spectroscopy, cells were directly measured in their culture medium, even if ionic. Figure 6 depicts the dielectric spectra (real and imaginary parts of relative permittivity) of the culture medium itself and a cell suspension of DoHH-2 cells line with a concentration of 100 million cells $/ \mathrm{ml}$. These cells serve as a biological model for blood cancer investigations. Owing to the high water content of cells and medium, the curves feature a relaxation frequency (maxima of $\varepsilon^{\prime \prime}$ around $15 \mathrm{GHz}$ ) close to that of water. The ionic content of the medium is noticeable at low frequency with the large increase of $\varepsilon$ " below $2 \mathrm{GHz}$. Both cells and medium spectra are significantly different and demonstrate the possible detection of cells in medium with microwaves from few $\mathrm{GHz}$ to $40 \mathrm{GHz}$.

As a supplement, the technique may also be utilized to quantify cells in suspension. Different concentrations have been evaluated, ranging from 100 million cells $/ \mathrm{ml}$ down to 17 million with the coplanar waveguide configuration shown in Fig.5. Repeated measurements are indicated at $10 \mathrm{GHz}$ in Figure 6 and exhibit an excellent reproducibility with a standard deviation less than 0.1 on both parameters $\left(\varepsilon^{\prime}\right.$ and $\left.\varepsilon^{\prime \prime}\right)[43]$.
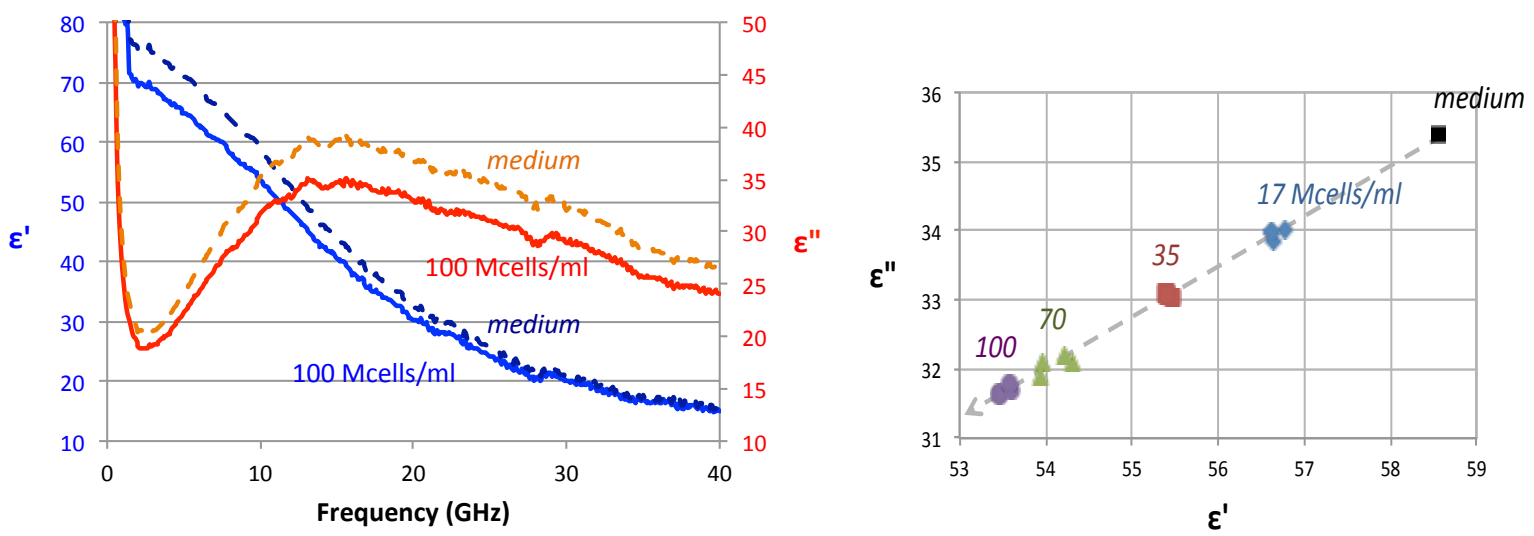

Figure 6. Complex Permittivity of RL human B lymphoma cell: dielectric spectra for 100 Mcells $/ \mathrm{ml}$ in their culture medium and dielectric chart @ $10 \mathrm{GHz}$ for cell concentrations ranging from 0 to $100 \mathrm{Mcells} / \mathrm{ml}$ [43].

Quantification of cells with microwave dielectric spectroscopy is particularly interesting for biomedical applications, as cell proliferation assessment is a key indicator in biological experiments. Impact of drugs is indeed evaluated by testing cell viability and consequently the cell's ability to duplicate.

As a supplement to the evaluation of cell populations, investigating the behavior of cells on an individual basis is also pertinent [18]. Single-cell measurements have therefore been made under different conditions (i.e. either static, in broadband [42] and narrowband [44], or in a flow [45]-[46]). 
For the broadband topology [42], the schematic of the microwave biosensor, which has been devised for this purpose, is shown in Figure 7. The corresponding picture featuring a trapped cell is part of Figure 5. It consists of a coplanar transmission waveguide with a capacitive gap located at the center of the sensor. Two tapers placed apart decrease the conductor size to an appropriate dimension commensurate with the cell. Thus, one can focus the electromagnetic fields onto the cell area. In this study, living human B lymphoma cells of the RL type, which constitute a well-known cell model for blood cancer investigations, were used. Perpendicular to the coplanar line, a microfluidic channel, equipped with a mechanical trap right above the capacitive gap, is inserted. This trap is used to locate the cell to be analyzed in the sensing area, while the channel maintains the living cell with all its required nutrients including salts, ions and proteins in its traditional medium during the experiments. The broadband single-cell sensor has been evaluated on a frequency range starting from $40 \mathrm{MHz}$ to $40 \mathrm{GHz}$. Several living cells in their culture medium have been individually tested and measurement results are given in Fig.7 for three different single RL lymphoma cells. The three measured capacitive contrasts yield a very similar spectrum. The dispersion between curves can be attributed to the intrinsic heterogeneity of the living cells and to the position of the cell in its proliferation cycle. The maximum capacitive contrast is obtained around $5 \mathrm{GHz}$ with a value close to $0.5-0.6 \mathrm{fF}$, which is much larger than the estimated measurement resolution of $0.01 \mathrm{fF}$.
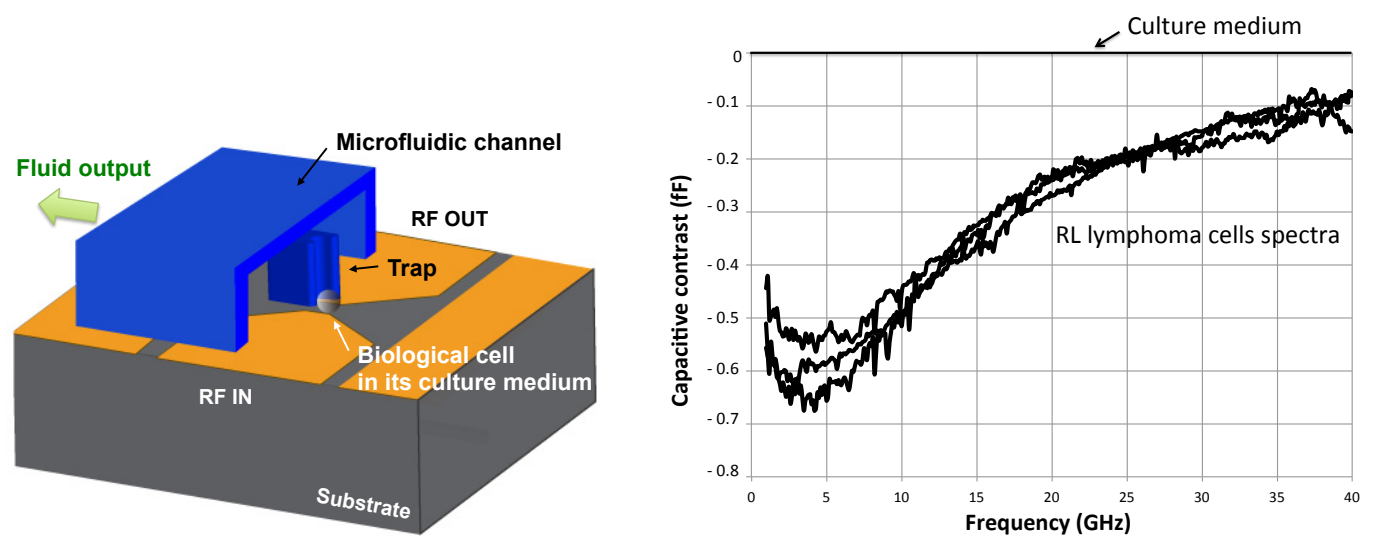

Figure 7. Schematic of the single-cell based microwave biosensor. Capacitive contrasts of human RL Iymphoma cells measured individually in their culture medium (RPMI with $10 \%$ of Foetal Calf Serum) from $40 \mathrm{MHz}$ to $40 \mathrm{GHz}$ [42].

As far as narrowband studies are concerned, interferometric configurations have been developed [44][46]. Single yeasts in De-lonized (DI) water have been evaluated in static with an interferometric circuit centered around $5 \mathrm{GHz}$, as indicated in Figure 8. The structure includes two branches, i.e. a reference and a "cell", within a microfluidic channel filled with DI water in one case and yeast in DI water in the other. To place the single yeast at the center of two tapered lines, a DC voltage is applied between the inlet and outlet of the "cell" fluidic channel. Several viable and non-viable yeast cells have been measured individually. The non-viable yeast cells have been obtained from the viable ones submitted to boiling for ten minutes and placed in DI-water. The transmitted scattering parameter versus frequency is given in Figure 8 . Viability of yeast may therefore be discriminated due to a frequency shift. Viable yeast cells present a lower resonant frequency than non-viable ones. 

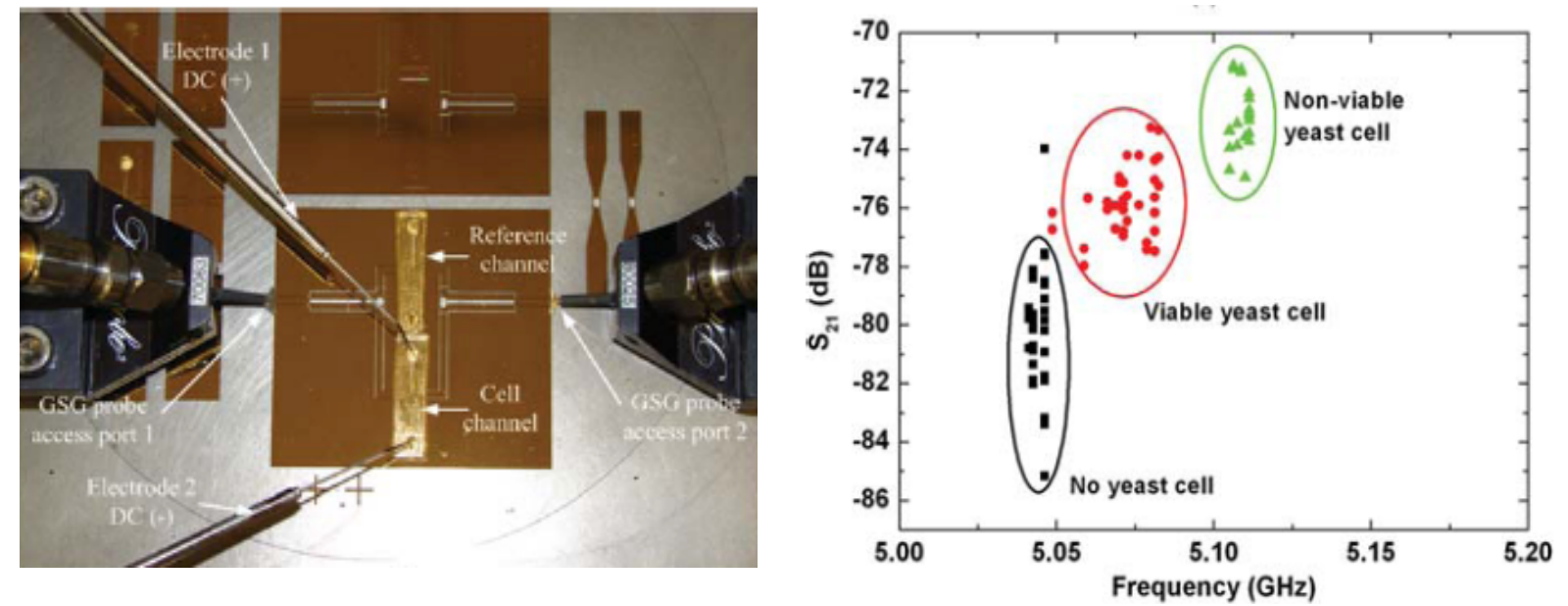

Figure 8. RF interferometer evaluated for yeast cells viability in de-ionized water [44].

A microwave interferometer was also defined at $1.6 \mathrm{GHz}$ for measurements in dynamic condition [45][46]. Single cells flowing in a microfluidic channel are detected while passing on top of an interdigitated electrode pair. The system may detect changes in capacitance of $650 \mathrm{zF}$ with a $50 \mathrm{~Hz}$ bandwidth. Yeast and Chinese hamster ovary cells in salt solution have been detected while passing on the sensing area.

The ability to detect cells individually in a flow paves the way for the development of RF cytometers, which may handle cells directly in their traditional medium and without preliminary preparation or labeling steps.

\section{Toward biological reaction observation and quantification}

Another key feature of microwave dielectric spectroscopy is the ability to evaluate biological reactions and to provide a quantification of the observed phenomenon.

As a first example, microwave dielectric spectroscopy has been studied to assess the cytoplasmic membrane permeabilization (and its consequence on the cells) of cells induced by chemo-treatment [47]. This process artificially kills the cells in a manner acceptable to biologists. Saponin, which is a chemical compound traditionally used to create pores into the cytoplasmic membrane of cells for intracellular labeling while maintaining the cell integrity, has been applied. Figure 9 shows a photography of the corresponding $\mathrm{DOHH}-2$ cells in their culture medium inside a coplanar waveguide as well as the dielectric contrasts of treated and untreated cells from $40 \mathrm{MHz}$ to $40 \mathrm{GHz}$.

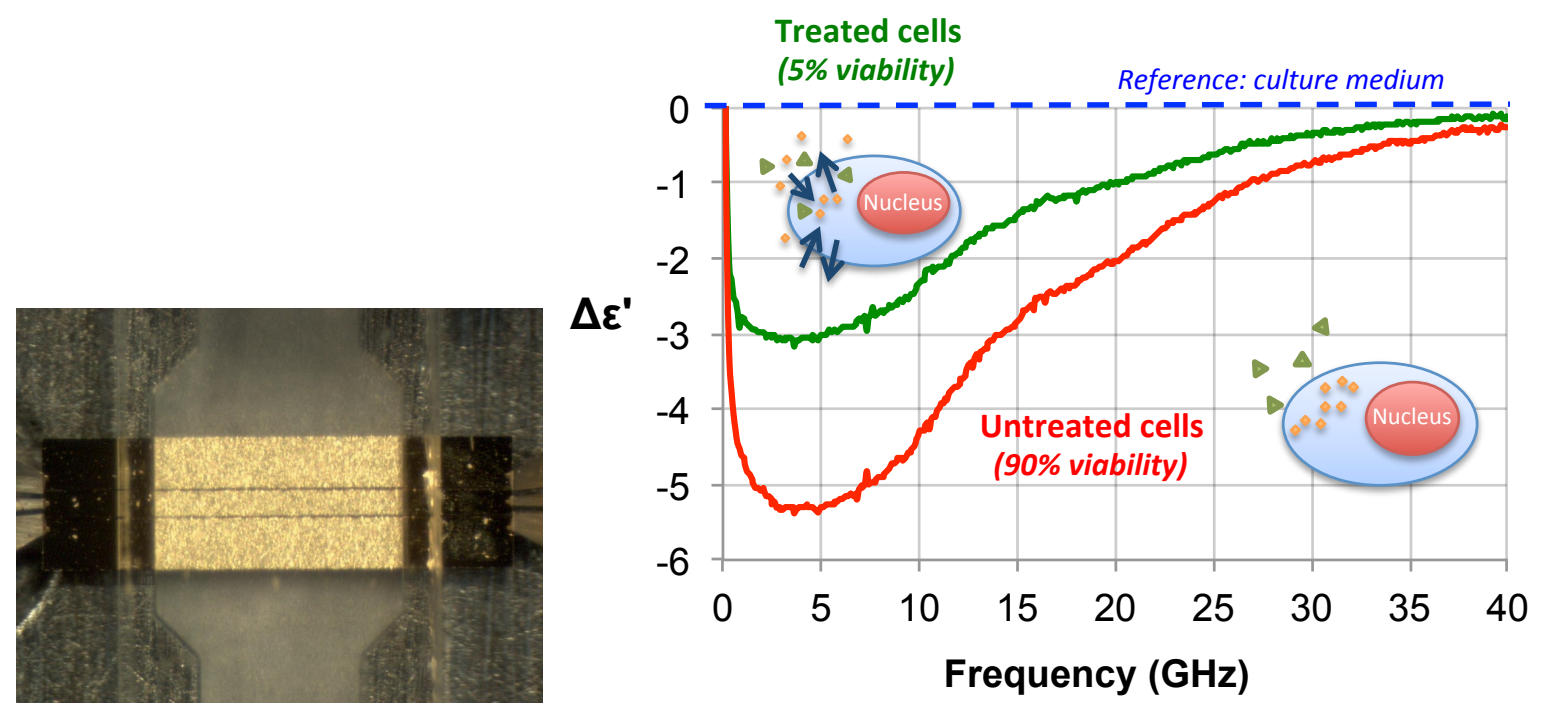

Figure 9. Picture of a coplanar waveguide loaded with DOHH-2 cells in suspension at the concentration of 100 Million cells $/ \mathrm{ml}$; dielectric contrast of treated and untreated cells with saponin for membrane permeabilization [47]. 
Viability of cell suspensions has been estimated with a biological technique using a marker. The treated and untreated cell suspensions exhibit viability values of $5 \%$ and $90 \%$ respectively. The saponin treatment on cell suspension tends to inhibit the dielectric contrast of cells in their medium due to the membrane permeabilization, which creates leakages of the cytosol in the surrounding culture medium with intra and extracellular exchanges of chemicals, as indicated in the inserted schematics of Figure 9. This results in a new solute equilibrium between internal cytosol and external medium. These measurements were repeated several times, at different days and with different sensors. An excellent measurement reproducibility has been secured and should convince biologists that microwave spectroscopy may significantly detect the viability of a cell population with an accuracy of $4 \%$.

The impact of the dimethylsulfoxide (DMSO) solvent has also been tested on the human embryonic kidney cell-line [36] using a reservoir placed on top of a coplanar waveguide. The cell suspensions were treated with $10 \%$ DMSO and then transferred to the CPW device for permittivity measurement. Realtime monitoring has been performed during thirty minutes. A progressive decrease in permittivity during time was demonstrated.
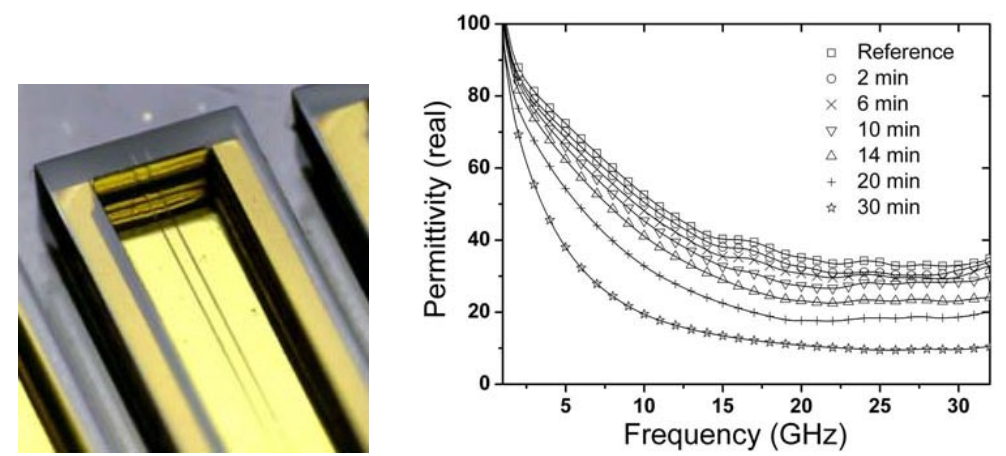

Figure 10. Realtime monitoring of DMSO impact on human embryonic kidney cell-line (HEK293) using a RF coplanar waveguide with an open reservoir [36].

This demonstrates the potential of the sensing technique for realtime monitoring.

\section{Toward microsystems integration for complete miniaturization}

All these results have been obtained using Vector Network Analyzers. Even if compact instruments are now marketed, they remain costly and cumbersome. To arrive at a high miniaturization with a complete lab-on-a-chip configuration, investigations aiming to integrate the analyzing part and not only the sensor are being conducted. A miniature VNA ranging from 14 to $16 \mathrm{GHz}$ for biomedical concentration measurements on RO4350 substrate was recently demonstrated. It includes a VCO centered around $15 \mathrm{GHz}$ with a four-way asymmetrical power divider, three I-Q mixers and a dual directional coupler. It was evaluated by connecting the VNA to a coplanar waveguide, which was loaded with several aqueous glucose solutions. Concentrations of glucose ranging from 0 to $10 \mathrm{~g} / \mathrm{dL}$ could be measured through normalized transmitted and reflection magnitude/phase [48]. Another configuration of VNA based on several six-port elements for multiband topology was also achieved [49]. Glucose solutions filled in a reservoir placed on top of coplanar waveguides with concentration ranging from 0 to $20 \mathrm{~g} / \mathrm{dl}$ were successfully evaluated at 6.6 and 19.7 and $32.4 \mathrm{GHz}$. The complete system configuration is indicated in Figure 11. The output voltages vary with glucose concentration. 

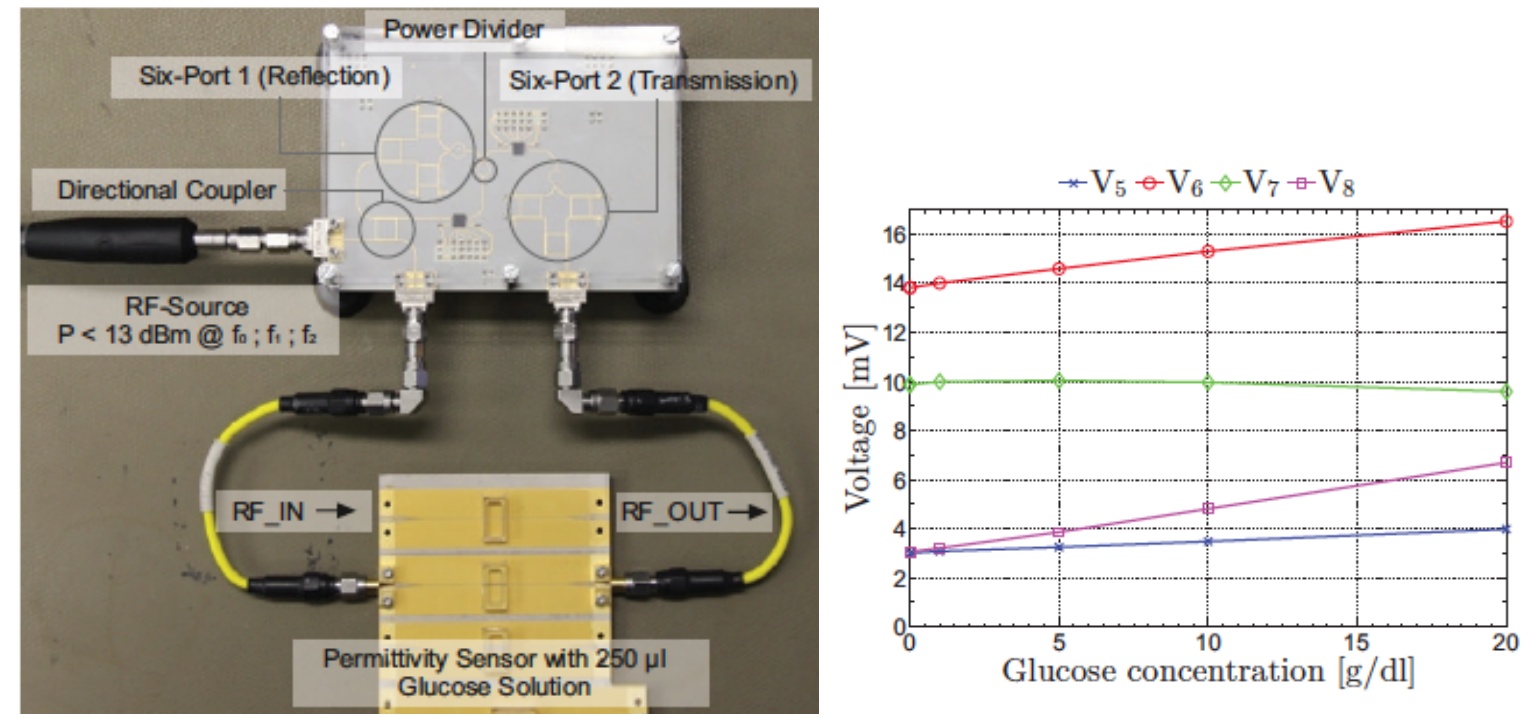

Figure 11. Multiband 2-port VNA [49].

In 2014, several CMOS based systems have been proposed for biological applications. An interferometric system with a $65-\mathrm{nm}$ CMOS technology, working at $6.5 \mathrm{GHz}$ and $17.5-\mathrm{GHz}$, was achieved with a sensitivity of $1.5 \mathrm{aF}$ [50]. Each sensing channel includes a VCO, two injection-locked oscillator sensors (instead of conventional LC resonators for signal-to-noise ratio enhancement), a mixer and a signal-conditioning analog front-end. Flowing polystyrene beads were successfully measured.

A CMOS dielectric spectroscopy system capable of extracting the complex permittivity of liquids from 0.62 to $10 \mathrm{GHz}$ was also presented [51]. It uses a configurable harmonic-rejection receiver to extract the complex permittivity, both real and imaginary parts with an rms error of less than $1 \%$ over the frequency band of $0.62-10 \mathrm{GHz}$. The liquid under test is placed on a sensing capacitor, which is embedded into a voltage divider topology with a fixed capacitor. The relative variations in magnitude and phase of the voltages permit to evaluate the real and imaginary parts of the broadband liquid permittivity, as indicated in Figure 12.
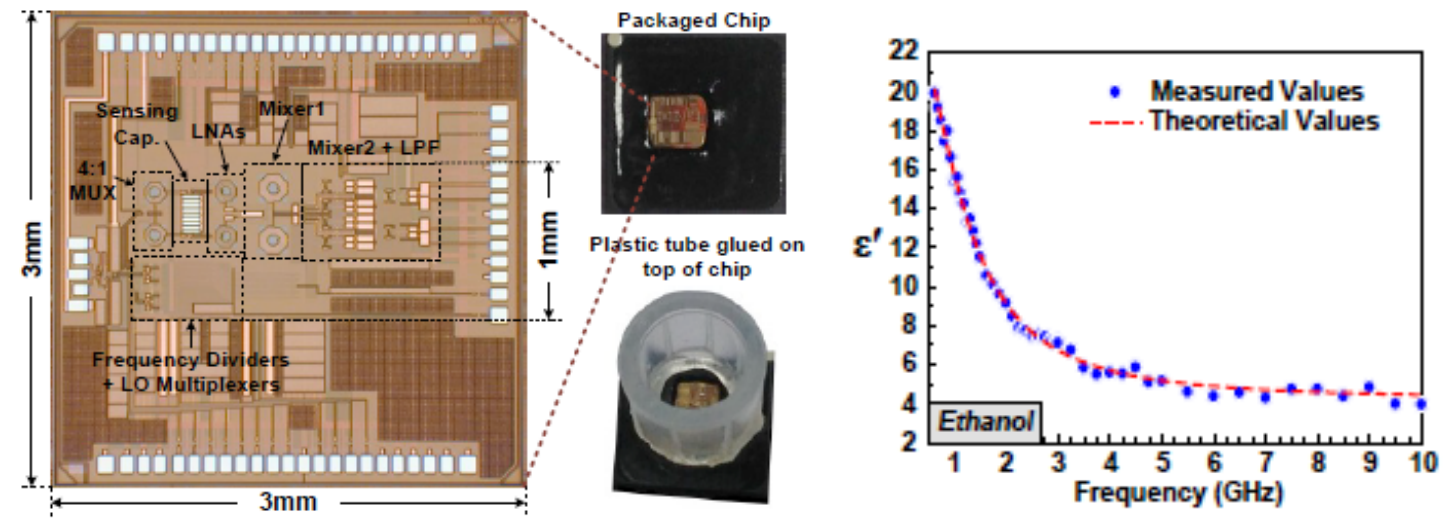

Figure 12. A $0.62-10 \mathrm{GHz}$ CMOS Dielectric Spectroscopy System for Chemical/Biological Material Characterization, validation with ethanol [51].

This demonstration does not include a sophisticated microfluidic setup at this stage. Combined with previously developed sensors capable of evaluation down to the single living cell, one may now consider the use of a complete and ultra compact microwave-based cellular analyzer.

\section{Conclusion : Miniature microwave spectroscopy is a promising technique for} intracellular analysis 
Given the huge development of microtechnologies, microwave dielectric spectroscopy can now be applied at the molecular and cellular levels, thus paving the way for new applications in the biological and medical domains. Circuits are indeed compatible with low biological volumes and cell size, and the frequency range supports intracellular analysis with the complete noninvasive penetration of electromagnetic waves into the living cell.

Key features have therefore been demonstrated such as the detection of cell population and single cells, with direct measurement in their culture medium, and the assessment of sensitivity to concentration and to cell state. Richness of the dielectric signature provides also a degree of specificity to the technique.

Miniature microwave spectroscopy can also be used for the realtime monitoring of biological reactions as well as high system integration. The specificities of microwave spectroscopy make this sensing technique a promising candidate for future cellular analyzing instruments alongside other established techniques such as fluorescent-based cell analyzers and microscopes and more recently those based on low frequency detection like the Xcelligence of Roche using tens of $\mathrm{kHz}$, which are more suitable for cell morphological and adhesion investigations. Advantages and drawbacks of these cellular analyzing techniques are therefore summarized in Table 1 in order to give a status of their actual capabilities. Applications for early diagnosis of various diseases as well as the preliminary evaluation of treatments directly on patient cells designed to improve the efficacy of therapies are the main objectives, which could be foreseen with microwave dielectric spectroscopy.

Table 1. Pros and cons of cellular analysis techniques

\begin{tabular}{|c|c|c|}
\hline & Pros & Cons \\
\hline Conventional optical techniques & $\begin{array}{l}\text { - High specificity with markers } \\
\text { - Precise images, molecular } \\
\text { resolution in cell } \\
\text { - Realtime monitoring }\end{array}$ & $\begin{array}{l}\text { - Cell preparation } \\
\text { - Invasivity in case of intracellular } \\
\text { labeling } \\
\text { - Time consuming } \\
\text { - Cost (resolution dependent) }\end{array}$ \\
\hline \multicolumn{3}{|l|}{ Impedancemetry } \\
\hline Low frequency & $\begin{array}{l}\text { - Noninvasivity for the cells } \\
\text { - Realtime monitoring } \\
\text { - Cost } \\
\text { - Potentially miniature }\end{array}$ & $\begin{array}{l}\text { - Not direct: low ionic medium } \\
\text { - Limited penetration of waves: } \\
\text { ionic diffusion and membrane } \\
\text { polarization related phenomena } \\
\text { - Global response of a cell, low } \\
\text { specificity compare to optical } \\
\text { techniques with markers }\end{array}$ \\
\hline Micro and milimeterwaves & $\begin{array}{l}\text { - Noninvasivity for the cells } \\
\text { - Direct, compatible with culture } \\
\text { medium of cells } \\
\text { - Realtime monitoring } \\
\text { - Intracellular measurement } \\
\text { - Global measurement of cells } \\
\text { - Cost } \\
\text { - Potentially miniature }\end{array}$ & $\begin{array}{l}\text { - Global measurement of cells, low } \\
\text { specificity compare to optical } \\
\text { techniques with markers }\end{array}$ \\
\hline
\end{tabular}

\section{Acknowledgements}

The authors would like to acknowledge the RITC foundation for financial support as well as the RENATECH network for supporting the microfabrication of some devices in LAAS-CNRS clean room, whose results have been presented in this article.

\section{References}

[1] C. Polk and E. Postow, Handbook of Biological Effects of Electromagnetic Fields. CRC Press, 1996, ch. 1 by K.R. Foster and H.P. Schwan. 
[2] H.P. Schwan, "Analysis of dielectric data: experience gained with biological materials," IEEE T. on Electrical Insulation, Vol. El-20, n6, Dec. 1985, pp. 913-922.

[3] A. Vander Vorst, A. Rosen, Y. Kotsuka, "RF/Microwave interaction with biological tissues," WileyInterscience, 2006.

[4] H. Fricke, "A mathematical treatment of the electric conductivity and capacity of disperse systems. I. The electric conductivity of a suspension of homogeneous spheroids," Phys Rev 24, pp. 575-587, 1924.

[5] K.S. Cole and R.H. Cole, "Dispersion and absorption in dielectrics. I. Alternating current characteristics," J. of Chemical Physics, vol. 9, pp. 341-351, 1941.

[6] S. Gabriel, R.W. Lau and C. Gabriel, "The dielectric properties of biological tissues", Physics in Medecine and Biology, vol. 41, no. 11, pp. 2231-2293, 1996.

[7] E.C. Fear, P.M. Meaney, M.A. Stuchly, "Microwave for breast cancer detection?" IEEE Potentials, pp. 12-18, Feb/Mar. 2003

[8] N.K. Nikolova, "Microwave Imaging for Breast Cancer," IEEE Microwave Magazine, vol. 12, pp. 7894, 2011.

[9] M. Lazebnik, D. Popovic, L. McCartney, C.B. Watkins, M.J. Lindstrom, J. Harter, S. Sewall, T. Ogilvie, A. Magliocco, T.M. Breslin, W. Temple, D. Mew, J.H. Booske, M. Okoniewski, S.C. Hagness, "A large-scale study of the ultrawideband microwave dielectric properties of normal, benign and malignant breast tissues obtained from cancer surgeries," Phys. Med. Biol., vol. 52, pp. 6093-6115, 2007.

[10] L. Sha, E.R. Ward, B. Stroy, "A review of dielectric properties of normal and malignant breast tissue," Proceedings of IEEE SoutheastCon 2002, pp. 457-462, 2002.

[11] A.P.O'Rourke et al., "Dielectric properties of human normal, malignant and cirrhotic liver tissue: in vivo and ex vivo measurements from 0.5 to $20 \mathrm{GHz}$ using a precision open-ended coaxial probe," Phys. Med. Biol. 52, pp. 4707-4719, 2007.

[12] W.M. Ahmed, S.J. Leavesley, B. Rjwa, M.N. Ayyaz, A. Ghafoor, J.P. Robinson, "State of the art of information extraction and quantitative analysis for multimodality biomolecular imaging, " Proc. of the IEEE, Vol. 96, March 2008, pp. $512-531$.

[13] G.M. Whitesides, "The origins and the future of microfluidics," Nature, vol. 442, pp. 368-373, 2006.

[14] F K Balagadde, et al., "Long-Term Monitoring of Bacteria Undergoing Programmed Population Control in a Microchemostat", Science, 309, 2005, pp.137-140.

[15] S. Grimmes, O. G. Martinsen, "Bioimpedance and bioelectricity basics", 2nd ed. Academic Press, 2008.

[16] X. Lu et. al. "Recent developments in single-cell analysis", Anal. Chim Acta 510, 127-138, 2004.

[17] P.J. Hung et. al., "Continuous perfusion microfluidic cell culture array for high-throughput cellbased assays", Biotechnol. Bioeng. 89, 1-8, 2005.

[18] D. Di Carlo, L.Luke, "Dynamic single cell analysis for quantitative biology", Anal. Chem. 79187925, Dec. 2006.

[19] S. Linström, H. Andersson-Svahn, "Overview of single-cell analyses: microdevices and applications", Lab Chip 10, 2010, pp. 3363-3372.

[20] S. S. Saliterman, "Fundamentals of BioMEMS and Medical Microdevices," Wiley-Inerscience, SPIE Press, Bellingham, Washington USA, 2006.

[21] N. Bao, J. Wang, C. Lu, "Recent advances in Electric Analysis of Cells in Microfluidic systems," Anal. Bioanal. Chem., vol. 391, pp. 933-942, 2008.

[22] Y.-B. Wang et. al., "A novel dual mode capacitor biosensor for real-time, label-free DNA detection", Int. Electron Devices Meeting, 2006. IEDM '06, 11-13 Dec. 2006.

[23] A. Han, et. al., "Whole Cell Electrical Impedance Spectroscopy For Studying Ion Channel Activity", 13th Int. Conf. on Solid-Sate Sensor, Actuators and Microsystems, Seoul, Korea, June 5-9, 2005.

[24] S. Gawad et. al., "Dielectric spectroscopy in a micromachined flow cytometer: theoretical and practical considerations, Lab Chip, vol. 4, pp. 241-251, 2004.

[25] T. Sun, H. Morgan, "Single-cell microfluidic impedance cytometry: a review," Microfluid. Nanofluid., vol. 8, pp. 423-443, 2010.

[26] D. Holmes, H. Morgan, "Single cell impedance cytometry for identification and counting of CD4 Tcells in human blood using impedance labels", Anal. Chem. 82, 1455-1461, 2010.

[27] H-B. Liu et. al., "Sensing minute change in biological cell monolayers with THz differential timedomain spectroscopy", Biosensors and Bioelectronics, Vol. 22, 2007, pp. 1075-1080.

[28] A. Treizebré et. al., "Investigation of living cells with a THz BioMEMS, 5th Int. Conf. on Microtech. in Medicine and Biology, April 2009, Quebec, Canada. 
[29] G.J. Wilmink, J.E. Grundt, "Invited review article: Current state of research on biological effects of Terahertz radiation," J. Infrared Milli Terahz Waves, vol. 32, pp. 1074-1122, 2011.

[30] S. Gabriel, R.W. Lau and C. Gabriel, "The dielectric properties of biological tissues", Physics in Medecine and Biology, vol. 41, no. 11, pp. 2231-2293, 1996.

[31] S. Mashimo, N. Miura, and T. Umehara, "The structure of water determined by microwave dielectric study on water mixtures with glucose, polysaccharides, and Lascorbic acid," J. Chem. Phys. 97, pp. 6759-6765, 1992.

[32] K. Fuchs, U. Kaatze, "Molecular dynamics of carbohydrate aqueous solutions. Dielectric relaxation as a function of glucose and fructose concentration," J. Phys. Chem. B, 105, pp. 2036-2042, 2001.

[33] K. Grenier, D. Dubuc, T. Chen, F. Artis, T. Chrétiennot, M. Poupot, J-J. Fournié, "Recent Advances in Microwave-based Dielectric Spectroscopy at the Cellular Level for Cancer Investigations," IEEE T-MTT, Vol. 61, Issue 5, Part 2, 2013, pp. 2023-2030.

[34] S.S. Stuchly, C.E. Bassey, "Microwave coplanar sensors for dielectric measurements," Meas. Sci. Technol., no. 9, 1998, pp. 1324-1329.

[35] G.R. Facer, D.A. Notterman et L.L. Sohn, "Dielectric spectroscopy for bioanalysis : from $40 \mathrm{~Hz}$ to $26.5 \mathrm{GHz}$ in a microfabricated wave guide," Applied Physics Letters, vol. 78, n 7, pp.906-908, 2001.

[36] S. Anghyun, T. Stintzing, I. Block, D. Pavlidis, M. Rieke, P. G. Layer, "High frequency wideband permittivity measurements of biological substances using coplanar waveguides and application to cell suspensions", 2008 IEEE MTT-Symposium International, pp. 915 - 918, 2008.

[37] J. Mateu, N. Orloff, M. Rinehart, J.C. Booth, "Broadband Permittivity of Liquids Extracted from Transmission Line Measurements of Microfluidic Channels," IEEE T-MTT-S 2007, pp. 523-526, 2007.

[38] K. Grenier, D. Dubuc, P-E. Poleni, M. Kumemura, H. Toshiyoshi, T. Fujii, H. Fujita, 'Integrated Broadband Microwave and Microfluidic Sensor dedicated to Bioengineering', IEEE Trans. on Microwave Theory and Techniques, Vol. 57, n²12, Dec. 2009, pp. 3246-3253.

[39] T. Chen, D. Dubuc, M. Poupot, J-J. Fournié, K. Grenier, "Accurate nanoliter liquid characterization up to $40 \mathrm{GHz}$ for biomedical applications: toward non-invasive living cells monitoring", IEEE T-MTT, Vol. 60, Issue 12, Part 2, Dec. 2012, pp. 4171-4177.

[40] James C. Booth, Nathan D. Orloff, Jordi Mateu, Michael Janezic, Matthew Rinehart, and James A. Beall, "Quantitative Permittivity Measurements of Nanoliter Liquid Volumes in Microfluidic Channels to $40 \mathrm{GHz}$ ", IEEE Transactions on Instrumentation and Measurements, Vol. 59, Dec. 2010, pp. 3279

[41] C. Dalmay, A.Pothier, P. Blondy, F. Lalloue, M.-O. Jauberteau, « Label Free Biosensors for Human Cell Characterization using Radio and Microwave Frequencies », IEEE MTT-S 2008.

[42] T. Chen, D. Dubuc, M. Poupot, J-J. Fournié, K. Grenier, "Microwave biosensor dedicated to the dielectric spectroscopy of a single alive biological cell in its culture medium", IEEE International Microwave Symposium, Seattle, USA, June 2013.

[43] F. Artis, D. Dubuc, J-J. Fournié, M. Poupot, K. Grenier, "Microwave dielectric bio-sensing for precise and repetitive living cells suspension analysis," IEEE EuMC 2013, Nuremberg, Germany, Oct. 2013, pp. 468-470.

[44] Y. Yang, P. Wang et al., "Distinguishing the viability of a single yeast cell with an ultra-sensitive radio frequency sensor, Lab on a Chip, 10, pp. 553-555, 2010.

[45] M. Nikolic-Jaric, S.F. Romanuik, G.A. Ferrier, G.E. Bridges, M. Butler, K. Sunley, D.J. Thomson, M.R. Freeman, "Microwave frequency sensor for detection of biological cells in microfluidic channels," Biomicrofluidics, 3, 034103, 2009.

[46] G.A. Ferrier, S.F. Romanuik, D.J. Thomson, G.E. Bridges, M.R. Freeman, "A microwave interferometric system for simultaneous actuation and detection of single biological cells," Lab Chip, 9, pp. 3406-3412, 2009.

[47] F. Artis, D. Dubuc, J-J. Fournié, M. Poupot, K. Grenier, " Microwave Dielectric Spectroscopy of Cell Membrane Permeabilization with Saponin on Human B Lymphoma Cells," IEEE IMS2014, Tampa, USA, June 2014.

[48] M. Hofmann, G. Fischer, R. Weigel, D. Kissinger, "Microwave-Based Noninvasive Concentration Measurements for Biomedical Applications," IEEE Trans. on MTT, Vol. 61, No. 5, May 2013, pp. 21952204.

[49] M. Hofmann, S. Linz, R. Weigel, G. Fischer, and D. Kissinger, "A Multiband 2-Port VNA for Biomedical Applications based on two Six-Port-Junctions," IEEE International Microwave Symposium, Seattle, USA, 2013 
[50] J.-C. Chien, M. Anwar, E.-C. Yeh, L. P. Lee, A. M. Niknejad, "A 6.5/17.5-GHz Dual-Channel Interferometer-based Capacitive Sensor in 65-nm CMOS for High-Speed Flow Cytometry," IEEE International Microwave Symposium, Tampa, USA, 2014.

[51] M.M Bajestan, A.A. Helmy, H. Hedayati, K. Entesari, "A $0.62-10 \mathrm{GHz}$ CMOS Dielectric Spectroscopy System for Chemical/Biological Material Characterization," IEEE International Microwave Symposium, Tampa, USA, 2014. 